\title{
Investigations on the Nature of Persistent Scatterers Based on Simulation Methods
}

\author{
Stefan Auer ${ }^{a}$, Stefan Gernhardt ${ }^{a}$, Richard Bamler ${ }^{a, b}$ \\ ${ }^{a}$ Remote Sensing Technology (LMF), Technische Universität München, Arcisstraße 21, 80333 München, \\ (Stefan.Auer, Stefan.Gernhardt)@bv.tum.de \\ ${ }^{b}$ Remote Sensing Technology Institute (IMF), German Aerospace Center (DLR), \\ Münchner Straße 20, 82234 Oberpfaffenhofen-Weßling, Richard.Bamler@dlr.de
}

\begin{abstract}
Using long-time coherent scatterers in SAR data, called Persistent Scatterers (PSs), the deformation signal of objects can be measured from space. However, the nature of the corresponding image signatures is not known in detail. Methods focused on the simulation of highly detailed object models help to understand the origin of PSs. In this paper, simulated SAR data are geometrically linked to PSs for one test site. Thereafter, single PSs are selected and identified in the simulated 3D model. The case study indicates that a high number of PSs lack of geometrical correspondence to building features. Moreover, it is shown that the often observed occurance of PSs located below ground level is not due to processing errors but is a systematic effect caused by fivefold reflection.
\end{abstract}

\section{INTRODUCTION}

Persistent Scatterer Interferometry (PSI) enables to measure the deformation signal of objects at the earth surface from space [1]-[3]. To this end, the phase of long-time coherent SAR image signatures is exploited. Very high resolution (VHR) PSI, using data of recent satellite missions like TerraSAR-X, TanDEM-X or Cosmo-SkyMed, enables to monitor single urban objects [4]. However, understanding the origin of PSs is still an open field of research. For instance, in [5] simulated data are imposed with Persistent Scatterers (PSs), but only single and double reflections of radar signals are considered. However, triple reflections are expected to be crucial for characterizing the signal response of urban objects, e.g. point patterns in case of buildings [6].

This paper aims at linking multiple reflections of radar signal with PSs. Real SAR data are automatically imposed with simulated signatures in order to confirm point signatures as strong building hints. Two SAR image signatures, being related to selected PSs, are identified in the $3 \mathrm{D}$ model of a test site. Thereby, to the knowledge of the authors, the directed analysis of PSs in three dimensions is conducted for the first time. Finally, the point signatures are localized in $3 \mathrm{D}$ in order to analyze the correspondance to building features.

\section{URban SCEnE OF InTEREST AND DATA SET}

As test site the center part of the main railway station of Berlin, Germany, is chosen. Figure 1 shows a perspective view onto the building complex. Two building components of cuboid form cross the rail tracks (building part 1 and building part 2). A roof made of glass, stabilized by metallic bows, links both building parts. Next to building part 2, a tower of triangular cross-section has been constructed. Both building parts 1 and 2 show regular structures as, for instance, vertical and horizontal bars made of metallic material can be found on each building floor (see Figure 2). The facade is composed by patches of glass which are connected by metallic stripes. Parts made of concrete can be seen through the glass patches between the horizontal iron bars.

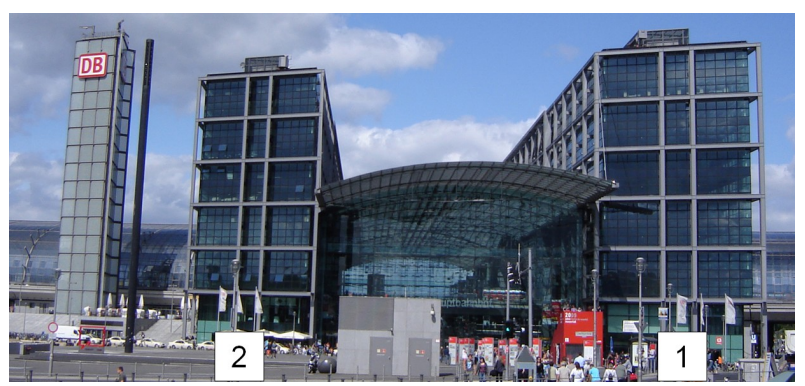

Fig. 1: Main railway station, Berlin. Two building components of cuboid form: building part 2 (left), building part 1 (right).
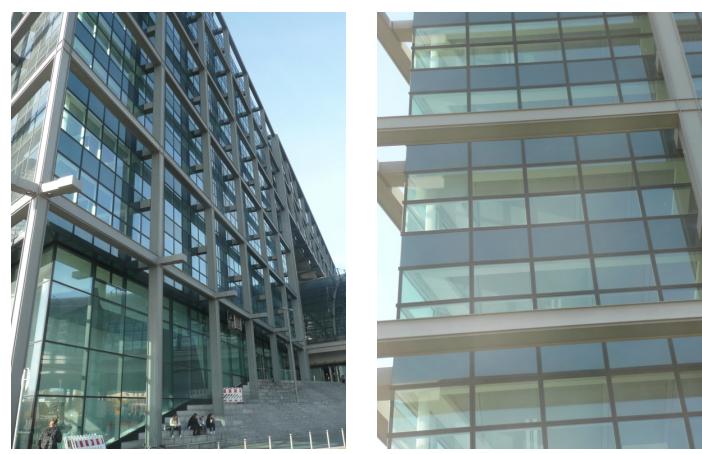

Fig. 2: Structure of main railway station, Berlin. Left: Horizontal and vertical bars surrounding the building. The facade is made of glass and partly covers concrete separating different floors of the building. Right: Glassy surfaces connected by metallic structures which cover parts of concrete.

20 high resolution TerraSAR-X spotlight data have been captured on a descending orbit with a local angle of incidence of $36.063^{\circ}$ and are processed using the PSI-GENESIS software of the German Aerospace Center (DLR). A temporal 
average image is presented in Figure 3. The spatial resolution of the image is $1.1 \mathrm{~m}$ in azimuth and $0.6 \mathrm{~m}$ in slant range, the pixel spacing is $0.433 \mathrm{~m}$ and $0.383 \mathrm{~m}$, respectively. Salient signatures on the SAR image may be considered as deterministic since random scattering is suppressed due to the averaging process.

In order to visualize SAR image signatures of interest, different clipping levels can be chosen. When clipping the intensity of the image at a low level, patterns of point signatures are visible as well as potentially diffuse signatures of low intensity (Figure 3a). When increasing the clipping threshold, the point signatures can be better localized (Figure 3b). Obviously, the strongest signal response is detected at the facades of building parts 1 and 2 .

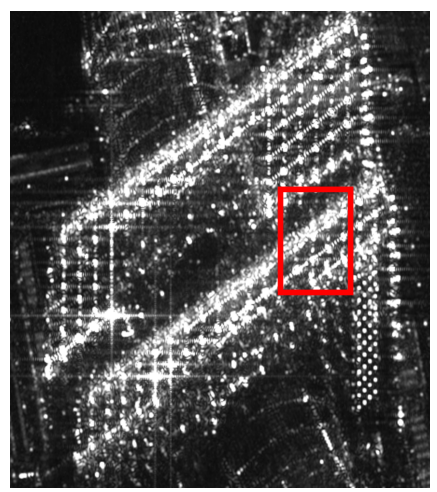

(a) Clipping at $3.4 \%$

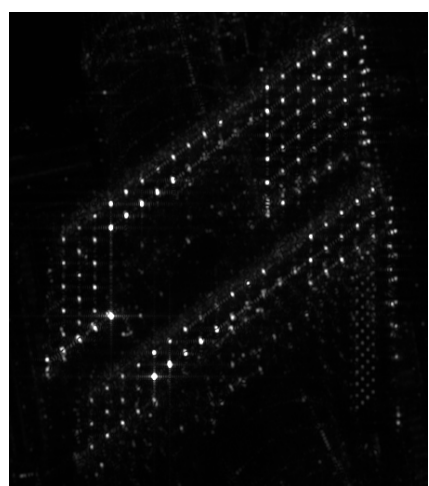

(b) Clipping at $34.7 \%$
Fig. 3: SAR temporal average images of the main railway station of Berlin, Germany. Red frame: area of interest for directed analysis of PSs (see Sections IV-A and IV-B). Ground range: top-down.

\section{SAR SIMULATION}

RaySAR, a 3D SAR simulator based on ray tracing methods [7], is used for providing artificial data in SAR geometry. To this end, a 3D building model is used which is based on architectural plans. While most of the building features are strongly generalized, the basic structure of facades is represented. Hence, the nature of PSs corresponding to reflection effects on the facades can be analyzed. A flat surface is defined beneath the above-ground parts of the railway station in order to represent the ground. Specular and diffuse reflection of the radar signal is enabled. As most of the surfaces show little roughness, the angular dependendance of diffuse reflection is considered.

For defining the surface characteristics, 6 object categories are defined: concrete and steel framework of building parts $1 / 2$, glassy roof parts, tower, metallic bows, building ground parts, and the ground. Building parts $1 / 2$ and the metallic bows are characterized by strong specular reflection and weak diffuse reflection. Glassy surfaces are expected to be mostly penetrated by the signal, i.e. the reflection capability is set very low. Medium diffuse reflection is assigned to the tower model as it is only composed by flat surfaces. However, the surface of the real tower is characterized by a high number of small corners.

When defining the virtual SAR sensor, the aspect angle with respect to north direction and the local angle of incidence are adapted to the master scene within the stack of SAR data. Sampling the model by means of ray tracing provides 4.68 million signal samples and lasts 20 minutes on a standard PC (2.0 GHz, $4 \mathrm{~GB}$ RAM). After ray tracing, reflectivity maps are provided whose sampling is adapted to the real SAR data.

\section{DIRECTED ANALYSIS OF PSS}

\section{A. Correspondence of SAR image signatures}

For comparing the simulated and real point signatures, the simulated reflectivity map has to be geometrically linked to the SAR image. To this end, the simulated point signatures can be used for distinguishing the railway station on the SAR image. The relative shift is determined by cross-correlating the reflectivity map with the SAR image section in frequency domain. Afterwards, the simulated maps can be imposed on the SAR image. Only non-zero grayvalues are accepted in order to conserve the background information of the real SAR image.

In the following, signatures are compared within a local area marked by a red frame in Figure 3. Salient point signatures are identified as being linked to triple bounce or to a combination of triple and fivefold bounce (Figure 4a). In contrast, the simulated signal contributions of bounce levels 2 and 4 are too weak to be distinguishable. They are of diffuse kind and are lost due to the angular dependence of diffuse reflection. In this regard, the necessary information for distinguishing 'specular' and 'diffuse' signal contributions is provided by RaySAR based on a geometrically analysis of each signal reflection. In Figure $4 \mathrm{~b}$, the distribution of PSs is shown. The number of PSs is much larger than the number of simulated signatures. The main reason is expected to be the limited level of detail of the building model as, for instance, the roof lacks of geometrical description.

The simulated positions of triple bounce contributions are expected to be reliable as the relative shifts with respect to the corresponding point signatures are small (Figure 4c). In contrast, pixels marked as fivefold bounce do not directly correspond to salient signatures but are located close to intensity peaks. The specular map, shown in Figure 4d reveals that almost all simulated signal contributions followed specular directions. For providing the map, the necessary information for the decision specular/non-specular has been derived during the ray tracing step. Based on a geometrical analysis of signal reflection, each signal bounce is marked in case of specular reflection. Thus, the occurrence of weak fivefold bounce signatures may be possible.

\section{B. Identification of PSs}

In the following, geometrical information provided by RaySAR is exploited for analyzing the nature of PSs. To this end, two signatures of interest are selected in the real SAR data 


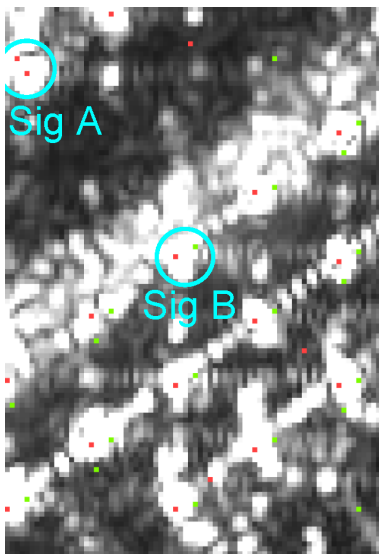

(a) Simulated signatures imposed on Figure 3a; red: triple bounce; green: fivefold bounce

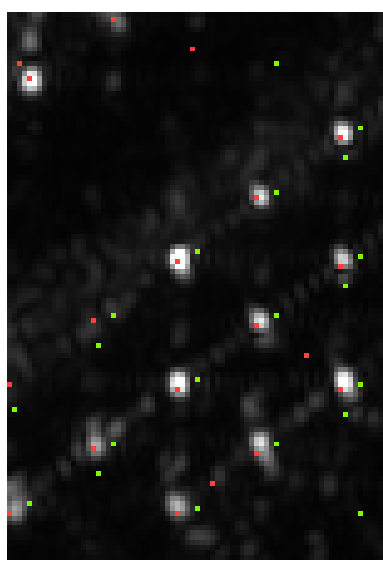

(c) Simulated signatures imposed on Figure 3b; red: triple bounce; green: fivefold bounce

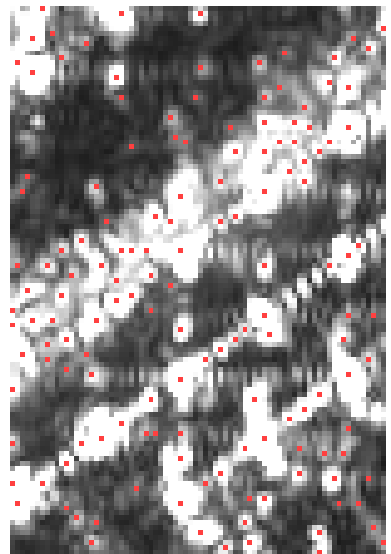

(b) PSs imposed on Figure 3a

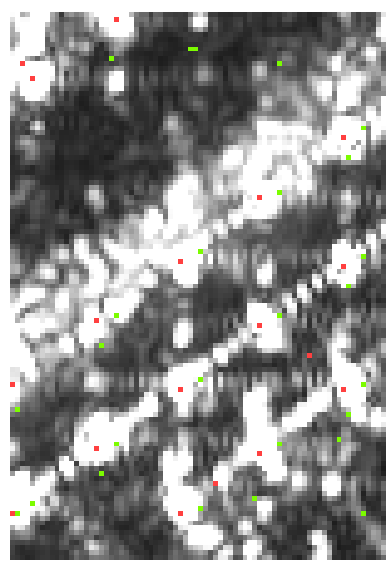

(d) Specular reflections imposed on Figure 3a; red: triple bounce; green: fivefold bounce
Fig. 4: Main railway station of Berlin: Signatures and PSs imposed on Figures 3a and 3b. Ground range: top-down.

(marked by circles in Figure 4a). Thereafter, the given link between the corresponding simulated signatures and the $3 \mathrm{D}$ building model is used for identifying the scatterers [8].

One strong PS and a nearby PS of medium strength represent signature A. On the simulated reflectivity map, strong specular triple bounce (signature A1) and a weak specular triple bounce signal peak (signature A2) are distinguishable.

Scatterer A1, corresponding to signature A1, is identified at building part 1 where the signal follows the path: vertical bar - facade - ground (and vice versa) or facade - vertical bar - ground (see Figure 5a). Both signal components are likely since the facade part interacting with the signal is located at the middle of two floors. In reality, this area is characterized by concrete covered with glass. As the glass is penetrated by the radar signal, the concrete is expected to be involved in the triple bounce effect.

Scatterer A2 is also located at building part 1 where the radar signal follows the path vertical bar - horizontal bar ground and horizontal bar - vertical bar - ground (see Figure
$5 b)$. This reflection phenomenon is linked to a specific kind of vertical bar whose end reaches beyond the end of the intersecting horizontal bar. Thus, a rectangular corner line is formed.

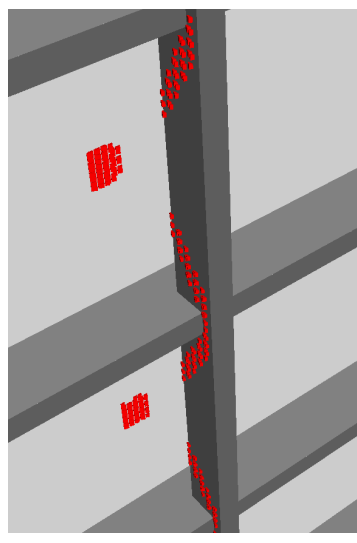

(a) Scatterer A1: triple bounce; signal paths: vertical bar (1) facade (2) - ground (3); facade (1) - vertical bar (2) - ground (3); signal reflection on ground not shown

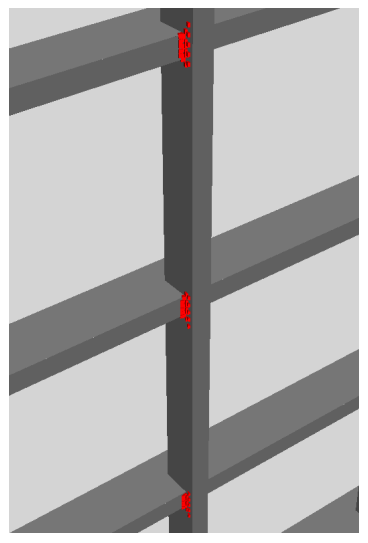

(b) Scatterer A2: triple bounce; signal paths: vertical bar (1) horizontal bar (2) - ground (3); signal reflection on ground not shown
Fig. 5: Identification of scatterers for signature A. Cube size: $5 \mathrm{~cm}$. Red: triple bounce.

At signature B one PS is selected. The simulation yields strong specular triple bounce (signature B1) and weak specular fivefold bounce (signature B2). Hence, as there is only one PS detected, the high intensity of signature B1 may mask the mainlobe of signature B2.

Scatterer B1 is identified at building part 2 where the radar signal follows the paths vertical bar - facade - horizontal bar, and facade - horizontal bar - vertical bar. In contrast, scatterer B2 occurs at building part 1 where the radar signal follows the paths ground - facade - bottom of horizontal bar - vertical bar - ground or ground - bottom of horizontal bar - facade vertical bar - ground. Again, the facade surface is the crucial component. Compared to the simulated result, the effective reflecting surface of the facade is reduced to a small horizontal band of concrete covered by glass. Nonetheless, the signature is visible in the real SAR data.

\section{C. $3 D$ position of simulated PSS}

As RaySAR provides data in azimuth, range, and elevation, the simulated signatures can be can be mapped into the $3 \mathrm{D}$ building model. In Figure 7, the signatures of type 'B1' are marked by white cubes on building part 1 . The pattern of point signatures is located on the facade but lacks of a geometrical link to specific building features. Thus, all triple bounce signals represent ghost corners.

The simulated fivefold bounce signatures (type: signature B2) are located beneath the ground level at building part 1 . On the left and center of Figure 8, two perspective views onto the resulting point pattern are shown. The spatial distribution depends to the structure of vertical and horizontal bars on 


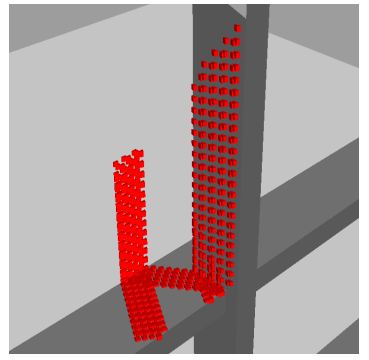

(a) Scatterer B1: specular triple bounce; signal paths: vertical bar (1) - horizontal bar (2) facade (3); horizontal bar (1) facade (2) - vertical bar (3)

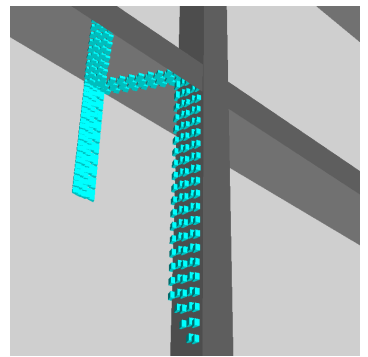

(b) Scatterer B2: specular fivefold bounce; signal paths: ground (1) - facade (2) - bottom of horizontal bar (3) vertical bar (4) - ground (5); ground (1) - bottom of horizontal bar (2) - facade (3) - vertical bar (4) - ground (5)

Fig. 6: Identification of scatterers for signature B. Cube size: $5 \mathrm{~cm}$. Red: triple bounce; Cyan: fivefold bounce.

the facade of building part 1. Likewise, some of the localized PSs are found beneath building part 1 (right of Figure 8). However, the points are less in numbers than in the simulated data and are irregularly distributed. A major factor influencing the deviation from the vertical plane is the limited accuracy of the localization in elevation due to the narrow orbital tube of TerraSAR-X. Moreover, the simulation is idealistic as the ground level is represented by a flat plane in the 3D model. In reality, the ground in front of the facade slightly varies in height and is partly covered with objects. Hence, the majority of the simulated fivefold bounce signal is unlikely to occur Nonetheless, regular patterns of PSs below the ground level are found for other isolated buildings in the center of Berlin. Hence, the simulation case study may give a strong hint that also specular fivefold bounce signatures correspond to PSs.

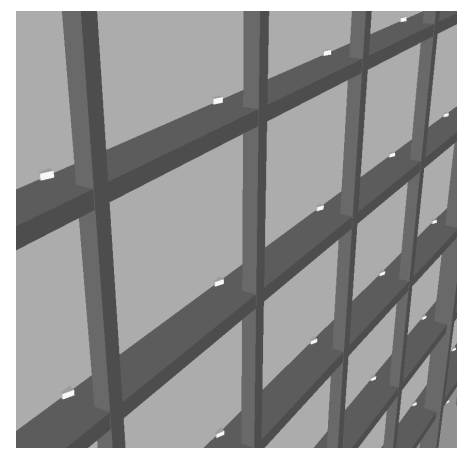

Fig. 7: Signatures of bounce level 3. Simulated signatures mapped on 3D building model (cube size: $20 \mathrm{~cm}$ ). All triple reflections represent ghost corners without correspondence to building features.

\section{CONCLUSION AND OUTLOOK}

For the main railway station of Berlin, simulated SAR image signatures of bounce levels higher than two have been linked to Persistent Scatterers. Thereafter, 3D SAR simulation
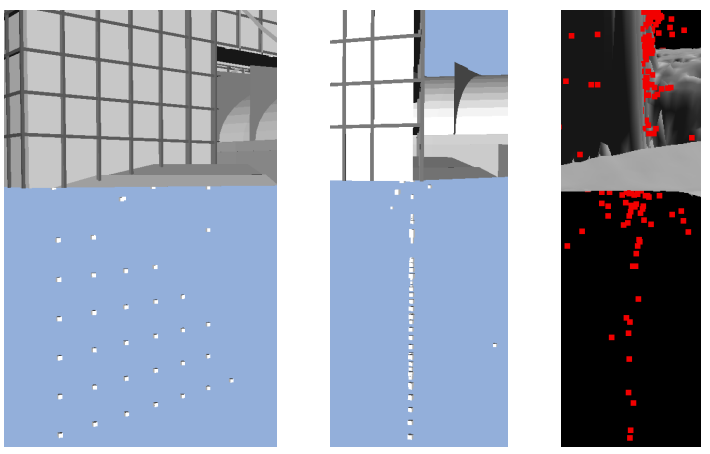

Fig. 8: Signatures of bounce level 5. Left and center: simulated signatures located beneath the ground level at building part 1 (cube size: $40 \mathrm{~cm}$ ). Right: PSs at building part 1 .

methods based on ray tracing have been used for identifying the corresponding scatterers in the 3D model. Eventually, the position of simulated $3 \mathrm{D}$ signatures has been compared to the position of PSs.

The simulation results confirm that specular triple reflections are crucial for the occurrence of PSs. For flat ground surrounding the building, specular fivefold bounce including the ground is considered as being relevant as well. All simulated signatures are related to ghost corners, i.e. there is no geometrical link to building features.

Future work will concentrate on preparing a-priori knowledge about urban objects for PSI. Moreover, as PSI only assumes one scatterer per resolution cell, the position of simulated signatures will be compared to results from SAR tomography.

\section{ACKNOWLEDGEMENT}

The 3D building model of the main railway station has been provided by the Berlin Business Location Center, State of Berlin, and was realized in a project financially assisted by the European regional development fund (ERDF).

\section{REFERENCES}

[1] A. Ferretti, C. Prati, and F. Rocca, "Permanent scatterers in SAR interferometry," IEEE Transactions on Geoscience and Remote Sensing, vol. 39, no. 1, pp. 8-20, Jan 2001.

[2] B. Kampes, Radar Interferometry - Persistent Scatterer Technique, F. van der Meer, Ed. Dordrecht, The Netherlands: Springer, 2006.

[3] A. Hooper, "Persistent scatterer radar interferometry for crustal deformation studies and modeling of volcanic deformation," Ph.D. dissertation, Stanford University, May 2006.

[4] S. Gernhardt, N. Adam, M. Eineder, and R. Bamler, "TerraSAR-X high resolution spotlight persistent scatterer interferometry," in Proceedings of FRINGE Workshop, 2009, p. on CD.

[5] A. Schunert, T. Balz, K. Liu, M. Liao, U. Soergel, and J. Wegner, "Simulation assisted high-resolution PSI analysis," in Proceedings of the ISPRS TC VII Symposium - 100 Years ISPRS, Vol. XXXVII, Part 7B, 2010.

[6] E. Michaelsen, U. Soergel, and U. Thoennessen, "Perceptual grouping for automatic detection of man-made structures in high-resolution SAR data," Pattern Recogn. Lett., vol. 27, no. 4, pp. 218-225, 2006.

[7] S. Auer, S. Hinz, and R. Bamler, "Ray-tracing simulation techniques for understanding high-resolution SAR images," IEEE Transactions on Geoscience and Remote Sensing, vol. 48, no. 3, pp. 1445 -1456, march 2010.

[8] S. Auer and R. Bamler, "3D analysis of trihedral reflection based on SAR simulation methods," in Proceedings of the European Conference on Synthetic Aperture Radar (EUSAR), Aachen, Germany, 2010, pp. 269272 